\title{
The Research of data migration algorithm and its application based on packet sets
}

\author{
Shanshan Kong ${ }^{1, a}$, Yongquan $\mathrm{Cai}^{2, \mathrm{~b}}$ and Haiyang $\mathrm{Yu}^{2, \mathrm{c}}$ \\ ${ }^{1}$ College of Computer Science, Beijing University of Technology, Beijing 100124, China; \\ ${ }^{2}$ College of Computer Science, Beijing University of Technology, Beijing 100124, China. \\ akongss27@163.com, ${ }^{\text {b }}$ cyq@bjut.edu.cn, ${ }^{\mathrm{c}}$ billyukiwi@163.com
}

Keywords: packet sets, dynamic characteristics, topological matrix, data migration.

\begin{abstract}
In order to meet the needs of user requirements and data transfer efficiency in the diversified outsourcing computing. Study the relationship between data migration order and Data storage topology, by the dynamic characteristics of P-sets. Through the analysis of the data transmission principle of network storage system and define the weights [d] of data migration, builds the dynamic adaptive data migration algorithm. And with a given topology matrix as the background shows the effectiveness of the approach.
\end{abstract}

\section{Introduction}

In set theory, a collection can be viewed as having certain properties of the object or individual constitute the overall, set $X_{1}$ and $X_{2}$ equal means that attribute sets $\alpha_{1}$ and $\alpha_{1}$ which determine $X_{1}$ and $X_{2}$ are completely the same. So when the attribute set $\alpha$ is fixed, the corresponding collection $X$ remains unchanged. According to the cantor set theory, we study the collection in the stationary state, however, the collection of real life and scientific and technological research tend to be dynamic.

Assumption [1] $X$ is a finite general set on $U, U$ is a finite element universe, $V$ is a limited attribute domain . $f \in F$ and $\bar{f} \in \bar{F}$ are element migrations, $F=\left\{f_{1}, f_{2}, \ldots, f_{\lambda}\right\}, \bar{F}=\left\{\bar{f}_{1}, \bar{f}_{2}, \ldots, \bar{f}_{\lambda}\right\}$ are element migration groups.

Definition[2] 1.1 Given a general set $X=\left\{x_{1}, x_{2}, \ldots, x_{m}\right\} \subset U$, and $\alpha=\left\{\alpha_{1}, \alpha_{2}, \ldots, \alpha_{k}\right\} \subset V$ is attribute set of $X, X^{\bar{F}}$ is called internal packet sets of $X$, called internal $P$ - sets for short, moreover

$$
X^{\bar{F}}=X-X^{-} \text {. }
$$

$X^{-}$is called $\bar{F}$ - element deleted set of $X$, moreover

$$
X^{-}=\{x \mid x \in X, \bar{f}(x)=W \notin X\} .
$$

If the attribute set $\alpha^{F}$ of $X^{\bar{F}}$ satisfies, $\alpha^{F}=\alpha \bigcup\left\{\alpha^{\prime} \mid \beta \in V, \beta \notin \alpha, f(\beta)=\alpha^{\prime} \in \alpha\right\}$.

Definition[2] 1.2 Given a general set $X=\left\{x_{1}, x_{2}, \ldots, x_{m}\right\} \subset U, \alpha=\left\{\alpha_{1}, \alpha_{2}, \ldots, \alpha_{k}\right\} \subset V$ is attribute set of $X, X^{F}$ is called outer packet sets of $X$, called outer $P$-sets for short, moreover

$$
X^{\bar{F}}=X \cup X^{+} \text {. }
$$

$X^{+}$is called $F$-element supplemented set $X$, moreover

$$
X^{+}=\left\{v \mid v \in U, v \notin X, f(v)=X^{\prime} \in X\right\} .
$$

If the attribute set $\alpha^{\bar{F}}$ of $X^{F}$ satisfies $\alpha^{\bar{F}}=\alpha-\left\{\alpha_{i} \mid \alpha_{i} \in \alpha, f\left(\alpha_{i}\right)=\beta \notin \alpha\right\}$.

Definition[3] 1.3 The set pair which are composed of internal $P$-sets $X^{\bar{F}}$ and outer $P$-sets $X^{F}$ are called $P$-sets (packet sets) generated by general set $X$, called $P$-sets for short, moreover

$\left(X^{\bar{F}}, X^{F}\right)$.

Where: general set $X$ is ground set of $\left(X^{\bar{F}}, X^{F}\right)$.

Theorem 1[1] If $\left(X^{\bar{F}}, X^{F}\right)$ is generated by set $X$, then

$$
X^{\bar{F}} \subseteq X \subseteq X^{F} \text {. }
$$


Definition [4] $1.4 \eta^{\bar{F}}$ is called internal package-degree of $X^{\bar{F}}$ about $X$, called internal degree of $X^{\bar{F}}$ for short, moreover

$$
\eta^{\bar{F}}=\operatorname{card}\left(X^{\bar{F}}\right) / \operatorname{card}(X) \text {. }
$$

Definition[5] $1.5 \eta^{F}$ is called outer package-degree of $X^{F}$ about $X$, called outer degree of $X^{F}$ for short, moreover

$$
\eta^{F}=\operatorname{card}\left(X^{F}\right) / \operatorname{card}(X) .
$$

Where: card=cardinal number, $\eta^{\bar{F}}, \eta^{F} \in R^{+}$.

Definition [6] 1.6 The set pair which are composed of internal degree of $X^{\bar{F}}$ and outer degree of $X^{F}$ are called package-degree of $P$ - sets $\left(X^{\bar{F}}, X^{F}\right)$, moreover

$$
\left(\eta^{\bar{F}}, \eta^{F}\right) \text {. }
$$

Obviously: $X$ is a finite general set on $U$, package-degree of $X$ satisfies $\eta=\operatorname{card}(X) / \operatorname{card}(X)=1$.

Theorem 2 (The theorem of dynamic internal $P$-sets) If $\alpha_{i}^{F}$ is a attribute set of internal $P-\operatorname{sets} X_{i}^{\bar{F}}(i=1,2, \cdots, n)$, satisfies

$$
\alpha_{1}^{F} \subseteq \alpha_{2}^{F} \subseteq \cdots \subseteq \alpha_{n}^{F} \text {. }
$$

Then

$$
X_{n}^{\bar{F}} \subseteq X_{n-1}^{\bar{F}} \subseteq \cdots \subseteq X_{1}^{\bar{F}} .
$$

Theorem 3 (The theorem of dynamic outer $P$-sets) If $\alpha_{i}^{\bar{F}}$ is a attribute set of internal $P$ - sets $X_{j}^{F}(j=1,2, \cdots, n)$, satisfies

$$
\alpha_{m}^{\bar{F}} \subseteq \alpha_{m-1}^{\bar{F}} \subseteq \cdots \subseteq \alpha_{1}^{\bar{F}} .
$$

Then

$$
X_{1}^{F} \subseteq X_{2}^{F} \subseteq \cdots \subseteq X_{m}^{F} .
$$

\section{P-Set with Its Data Screening Characteristics}

Assumption In discussion of the next sets $X^{\bar{F}}, X, X^{F}$ are called data, $X^{\bar{F}}, X, X^{F}$ denoted as $[x]^{\bar{F}}$, $[x],[x]^{F}$ or $[x]^{\bar{F}}=X^{\bar{F}},[x]=X,[x]^{F}=X^{F}$.

Definition 2.1 $[x]=\left\{x_{1}, x_{2}, \cdots x_{q}\right\} \subset U$ is called data, $x_{i} \in[x]$ is called data element of $[x], i=1,2, \ldots, q$; If $[x]$ has attribute set $\alpha$, moreover

$\alpha=\left\{\alpha_{1}, \alpha_{2} \cdots \alpha_{k}\right\}$.

Definition 2.2 Record $[d]$ is the right value of data $[x]$, moreover

$[d]=\left\{d_{1}, d_{2}, \cdots d_{m}\right\}$.

If $d_{i} \in R$ are the right value of $x_{i} \in[x], R$ is real number set, $x_{i}$ are data elements of $[x]$.

Definition 2.3 Data $[x]^{F}$ is called outer data of $[x]$, moreover

$$
[x]^{F}=\left\{x_{1}, x_{2}, \cdots x_{r}\right\} \text {. }
$$

If the attribute set $\alpha^{\bar{F}}$ of $[x]^{F}$ and the attribute set $\alpha$ of $[x]$ satisfies

$$
\alpha^{\bar{F}}=\alpha-\left\{\alpha_{i} \mid \bar{f}\left(\alpha_{i}\right)=\beta \notin \alpha, \bar{f} \in \bar{F}\right\} .
$$

Where: $\alpha_{i}$ satisfies $\alpha_{i} \in \alpha, \bar{f} \in \bar{F}$ which changes $\alpha_{i}$ into $\bar{f}\left(\alpha_{i}\right)=\beta_{i} \bar{\epsilon} \alpha$.

Definition $2.4 x_{0}$ is called initial data element of $[x]$, moreover

$$
x_{0}=\bigcap_{i=0}^{n}[x]_{i}^{F}
$$

Definition 2.5 $[X]_{0}^{F}$ is called initial data, moreover

$$
[x]_{0}^{F}=\left\{x_{0}\right\} \text {. }
$$


Definition $2.6[x]_{*}^{F}$ is called terminate data, moreover $\operatorname{card}\left([X]_{*}^{F}\right)=\operatorname{card}(U)$.

Existence theorem of outer data If $\alpha$ is the attribute set of data $[x], \forall \alpha_{i} \in \alpha, \exists \bar{f} \in \bar{F}$ which changes $\alpha_{i}$ into $\bar{f}\left(\alpha_{i}\right)=b_{i} \notin \alpha$, there exists data $[x]^{0}$ whose attribute set is $\alpha^{\bar{F}},[x]^{0}$ is a outer data of $[x]$, moreover

$[x]^{O}=[x]^{F}$.

Where: $\alpha^{F}=\alpha-\left\{\alpha_{i} \mid \bar{f}\left(\alpha_{i}\right)=b_{i}\right\}$.

Proof: For $\alpha^{F}=\alpha-\left\{\alpha_{i} \mid \bar{f}\left(\alpha_{i}\right)=b_{i}\right\}$,from the dependence relationship between attributes and data, it can know that there exists data $[x]^{*}$ whose attribute set is $\alpha^{F}$; Because $\alpha^{F}=\alpha-\left\{\alpha_{i} \mid \bar{f}\left(\alpha_{i}\right)=b_{i} \notin \alpha\right\}$ and literature [1,2,3] it gets that: data $[x]^{0}$ and $[x]$, which has attributes set $\alpha^{F}$ and $\alpha$ respectively, satisfies $[x] \subseteq[x]^{0},{ }^{[x]^{0}}$ is a outer data of $[x]$. Thus, existence theorem of hidden data can be obtained.

According to proposition 1, when determining whether the maximum terms clause can be expanded by the clauses, we should determine whether it can be expanded by the clauses of smaller degree first. In the following, the algorithm determining which satisfied the partial maximum terms space will be given.

Equations should be typed within the text, centred, and should be numbered consecutively throughout the text. They should be referred to in the text as Equation (n). Their numbers should be typed in parentheses, flush right, as in the following example.

Step $1 U$ is divided into two subsets $[x]^{F}$ and $[x]^{\bar{F}}$. Initial time, $[x]_{0}^{F}=\left\{x_{0}\right\},[x]^{\bar{F}}=U-[x]^{F}$;

Step 2 Calculate the value $d(x)$ of each element $x$ in the collection $[x]^{\bar{F}}$, according to the value of $d(x)$ to find the shortest distance $\mathrm{x}_{0}$ node $x$ from $[x]^{\bar{F}}$;

Step 3 Reset $[x]_{i}^{F}$ to $[x]_{i-1}^{F} \cup\{x\}$, reset $[x]_{j}^{\bar{F}}$ to $[x]_{j-1}^{\bar{F}_{1}}-\{x\}$, if $\operatorname{card}[x]^{\bar{F}}=0$, then stop, otherwise repeat 2;

Step 4 Test $\operatorname{card}\left([x]_{0}^{F}\right)=\operatorname{card}(U)$.

Where, $d(x)$ indicate that the shortest path length from ${ }^{x_{0}}$ to ${ }^{x}$ and do not contain other nodes of $[x]^{\bar{F}}$.

\section{Application of P-Sets in Resistance}

The heating device resistance users always hope to use the shortest time to obtain the required quantity of heat, which requires the resistance faces enormous challenges hitherto unknown, and the most important is to find the optimal resistance selection and realization method. Then screening step by step, screening out the shortest path of every node to the starting point, until all the points are chosen.

Table 1 Resistance weights matrix

$$
\left[\begin{array}{cccccc} 
& x_{0} & x_{1} & x_{2} & x_{3} & x_{4} \\
a_{0} & 0 & 3 & - & - & 10 \\
a_{1} & 3 & 0 & 4 & - & 8 \\
a_{2} & - & 4 & 0 & 5 & 7 \\
a_{3} & - & - & 5 & 0 & 6 \\
a_{4} & 10 & 8 & 7 & 6 & 0
\end{array}\right]
$$


The background characteristic of this example is that the attribute of data $[x]=\left\{x_{1}, x_{2}, \cdots x_{k}\right\}$ is $\alpha=\left\{\alpha_{1}, \alpha_{2}, \cdots \alpha_{n}\right\}$. Among them, the data element $x_{i}$ on behalf of the name of the routing, $\alpha_{i}$ represents the $i$ routing, $d_{i}$ represents the value of the $i$ data element in $[x]^{\bar{F}}$ with new data elements and data in $[x]^{F}$. Initial state data $[x]^{F}=\left\{x_{0}\right\}, \quad[x]^{\bar{F}}=\left\{x_{1}, x_{2}, \cdots x_{4}\right\}$, there are $\alpha_{i}$ transfers out from $\alpha=\left\{\alpha_{1}, \alpha_{2}, \cdots \alpha_{n}\right\}$, whenever the data to select a route point ,there exist attribute migrations $\bar{f} \in \bar{F}$, set $\alpha^{\bar{F}}=\alpha-\left\{\alpha_{i} \mid \bar{f}\left(\alpha_{i}\right)=\beta \notin \alpha, \bar{f} \in \bar{F}\right\}$. At this point, $\operatorname{card}\left([x]^{F}\right)$ become larger, $\operatorname{card}\left([x]^{\bar{F}}\right)$ become smaller. This continues until the $\operatorname{card}\left([x]^{\bar{F}}\right)=0$. Using the screening steps of $P$ - sets and routing weights matrix given in the above part to get below five data tables, data from the initial route to each route path selection process and results.

Table 2 Dynamic adaptive data migration algorithm

\begin{tabular}{lcccccc}
\hline \multicolumn{1}{c}{$[X]^{F}$} & $x$ & $d(x)$ & $d\left(x_{1}\right)$ & $d\left(x_{2}\right)$ & $d\left(x_{3}\right)$ & $d\left(x_{4}\right)$ \\
\hline$\left\{x_{0}\right\}$ & 0 & 3 & - & - & - & 10 \\
$\left\{x_{0}, x_{1}\right\}$ & $x_{1}$ & 3 & 3 & 7 & - & 11 \\
$\left\{x_{0}, x_{1}, x_{2}\right\}$ & $x_{2}$ & 7 & 3 & 7 & 12 & 11 \\
$\left\{x_{0}, x_{1}, x_{2}, x_{3}\right\}$ & $x_{3}$ & 12 & 3 & 7 & 12 & 11 \\
$\left\{x_{0}, x_{1}, x_{4}\right\}$ & $x_{4}$ & 11 & 3 & 7 & 12 & 11 \\
\hline
\end{tabular}

\section{Summary}

In the ordinary set theory, elements and relations among sets are identified, and cardinal number of a set is the same. It has static characteristics. But in the actual problem, only a few sets have the static characteristics, most of sets have dynamic feature. Such as: Dynamic information system, Moving target images, Dynamic data system and so on. These issues have common characteristic that is "dynamic." $P$ - sets are new mathematical model to study dynamic data system and dynamic information systems, $P$ - sets have great use of space, especially in computer science and information science. In fact, $P$ - sets had been used in information science, system science to solve the many application problems by scholars.

\section{References}

[1] Shi, K.Q. "P-sets and its applications", An International Journal Advances in Systems Science and Application, 9(2), pp. 209-219 (2009).

[2] Shi, K.Q. "P-sets”, Journal of Shandong University (Natural Science), 43(11), pp. 77-84 (2008).

[3] Shi, K.Q. "Internal P-sets and data outer-recovery”, Journal of Shandong University (Natural Science), 44(4), pp. 8-14 (2009).

[4] Tang, J.H., Chen, B.H., Shi, K.Q. "P-sets and (F,F) -data generation-identification”, Journal of Shandong University (Natural Science), 44(11), pp. 83-92 (2009).

[5] Yu, X.Q. "Identification and filtering of P-sets", Journal of Shandong University (Natural Science), 45(1), pp. 94-98 (2010).

[6] Yu, X.Q. "Area characteristic of P-sets. Journal of Shandong University (Natural Science)”, 45(2), pp. 99-102 (2010). 